\title{
Critical Overview of the Use of Tethered Bilayer Lipid Membranes with Electrochemical Techniques
}

\author{
Brianna D Murphy and Angel A.J. Torriero* \\ School of Life and Environmental Sciences, Deakin University, Burwood, Victoria
} 3125, Australia

*Corresponding author: Angel AJ Torriero, School of Life and Environmental Sciences, Deakin University, Burwood, Victoria 3125, Australia, Email: angel.torriero@deakin.edu.au

\section{Mini Review \\ Volume 2 Issue 1}

Received Date: May 01, 2018

Published Date: June 19, 2018

\section{Abstract}

Studying various substrate interactions with the plasma membrane is difficult due to the large variety of molecules that compose the biological membranes. The importance of understanding plasma membrane interactions, behaviour and properties prompted the development of artificial membranes to recapitulate fundamental aspects of membrane biology. Tethered bilayer lipid membranes (t-BLM) are a versatile type of artificial membrane that enables the simulation of real biological membranes in a controlled, stable environment. Numerous studies have uncovered valuable data using t-BLMs but there are still some unknowns related to t-BLM research that has been overlooked. For example, the effect of unsaturation in phospholipid tails is one of these unknowns. By focusing on gaps in t-BLM research and Electrochemical Impedance Spectroscopy, this manuscript makes evident that further development and classification of t-BLMs is still required to make them a more sound biological mimic.

Keywords: Plasma membrane; Tethered bilayer lipid membranes; Phospholipid tails; Electrochemical Impedance Spectroscopy.

Abbreviations: PC: Phosphotidylcholine; PE: Phosphatidylethanolamine; PS: Phosphatidylserine; SM: Sphingomyelin; ICS: Ion Channel Switch; BLM: Black Lipid Membrane; s-LBM: Supported Lipid Bilayer Membrane; h-BLM: hybrid Bilayer Lipid Membrane; tBLM: tethered Bilayer Lipid Membrane; SAM: SelfAssembled Monolayer; PTE: 1,2-dipalmitoyl-sn-glycero3-phosphothioethanol; EIS: Electrochemical Impedance Spectroscopy; AMPs: Antimicrobial Peptides.

\section{Introduction}

The term 'cell', coined by Robert Hooke in 1665, ignited the several century journey of studying prokaryotic and eukaryotic cells and their diverse components [1]. The phospholipid bilayer is one of the major components of cells that form a continuous barrier around cells separating the interior and exterior environments [2]. Figure 1 shows a cartoon of a cellular membrane representing some of the biological molecules that are embedded into the phospholipid bilayer, such as proteins, lipids and carbohydrates; making it a very complex and strictly regulated system [3].

Besides the imperative role of compartmentalization, phospholipid membranes are involved in many exchange processes between the internal and external cellular environments. Due to the integral functions of selective permeability, the phospholipid bilayer is the host site for many pharmaceuticals [4]. The excellent biocompatibility and amphiphilicity of the phospholipid bilayer makes passive diffusion through the membrane the most common route of pharmaceutical delivery and therefore a primary research target [5]. Although biological membranes are crucial to almost all cellular phenomena and have been intensively studied over the 


\section{Medicinal \& Analytical Chemistry International Journal}

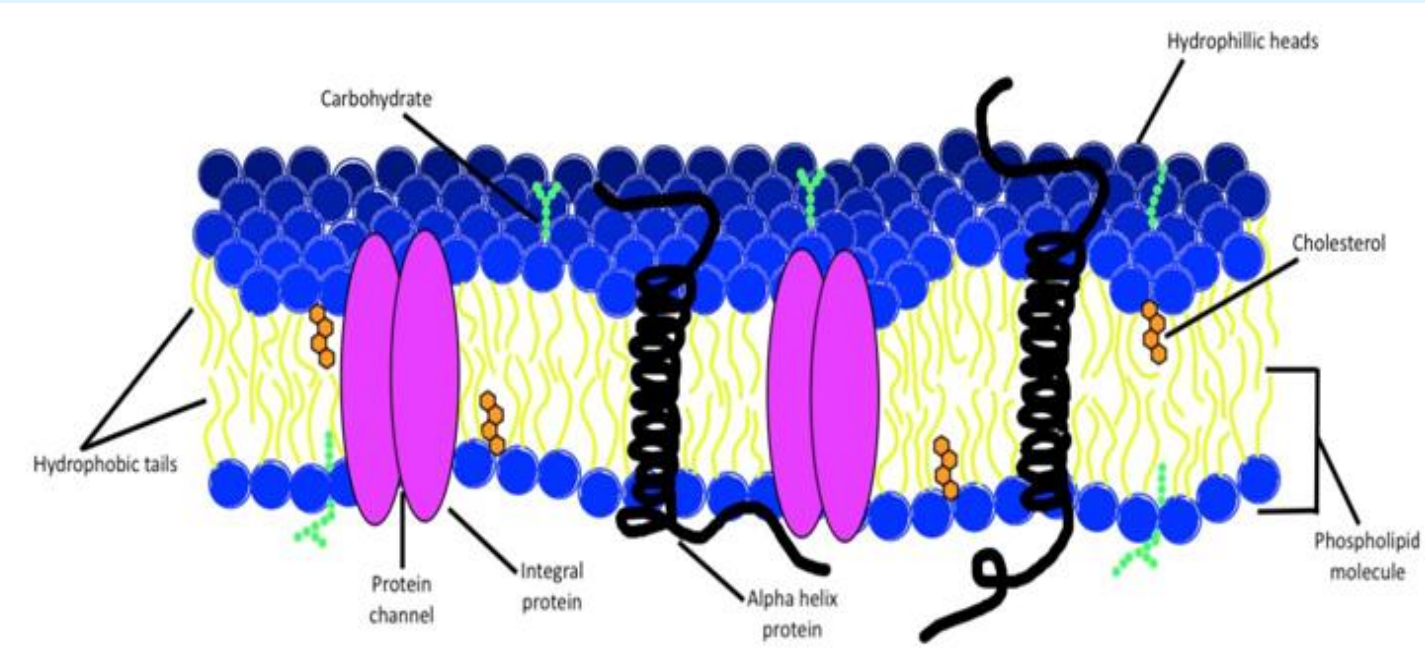

Figure 1: Schematic illustration of a plasma membrane demonstrating some of the complexities embedded within including carbohydrates, proteins and lipids.

last 40 years, our understanding of phospholipid membranes is still rudimentary [4]. A more thorough understanding of purely the phospholipid bilayer at a molecular level without its complexities still needs to happen [6].

Depending on the alcohols contained in the phospholipids they can be divided into glycerophospholipids and sphingolipids [7]. Sphingolipids are important components of neuronal membranes but glycerophospholipids are more abundant in animal cell membranes [8]. The dynamic amphiphilic structure that causes the phospholipids to aggregate and spontaneously self-assemble into bilayers is commonly exploited throughout membrane studies [9]. The hydrophilic head group and hydrophobic fatty acid tails are variables for different types of phospholipids. There are four major types of phospholipid heads that comprise the plasma membrane of mammalian cells: phosphatidylcholine (PC), phosphatidylethanolamine

$(\mathrm{PE})$, phosphatidylserine (PS) and sphingomyelin (SM) [10]. But, PC is the most abundant glycerophospholipid in eukaryotic phospholipid membranes [11].

The complexity of biological membranes and their interactions with the intracellular and extracellular networks make their direct investigation difficult and particularly restrict to in- situ studies of the lipid bilayer environment. Therefore, the direct study of just the phospholipid membrane as a model system of real cell membranes requires creation of rational models, which recapitulate fundamental aspects of the cell membranes structure and function. Artificial membranes enable composition tuning, making it possible to start with the phospholipid bilayer followed by slowly introducing membrane components to investigate their contribution to the cell. These models have vasty contributed to the advancement of biotechnology in the field of healthcare, environmental monitoring and energy storage [12]. Ion Channel Switch (ICS) biosensors used in pathogen detection [13,14], diffusion controlled drug delivery membranes $[15,16]$ and dialysis membranes [17] barely scratch the surface of artificial membrane applications.

Various methods have been developed to form different types of artificial lipid membranes including black lipid membrane (BLM), supported lipid bilayer membrane (s-BLM), hybrid bilayer lipid membrane (hBLM) and tethered bilayer lipid membrane (t-BLM) [18]. BLMs were developed over 40 years ago and have substantially contributed to the current understanding of biological membranes. Unfortunately, the freestanding planar film that constitutes the BLM causes such an unstable lifetime ( $<1$ hour) making it an outdated method compared to the s-LBM [19]. Solid substrates are often used to support the s-LBM, providing mechanical stability to allow for vigorous experimentation including electrochemical techniques [12]. Various materials can serve as membrane supports such as gels, mica, ceramics, silicon or noble metals [6]. The primary limitation of supported bilayers is the extensive hydrodynamic coupling between the bilayer and substrate resulting in a lower diffusion coefficient [20]. To circumvent this problem t-BLM are used as they don't encounter substrate compatibility limitations that affect the diffusion coefficient [21]. 


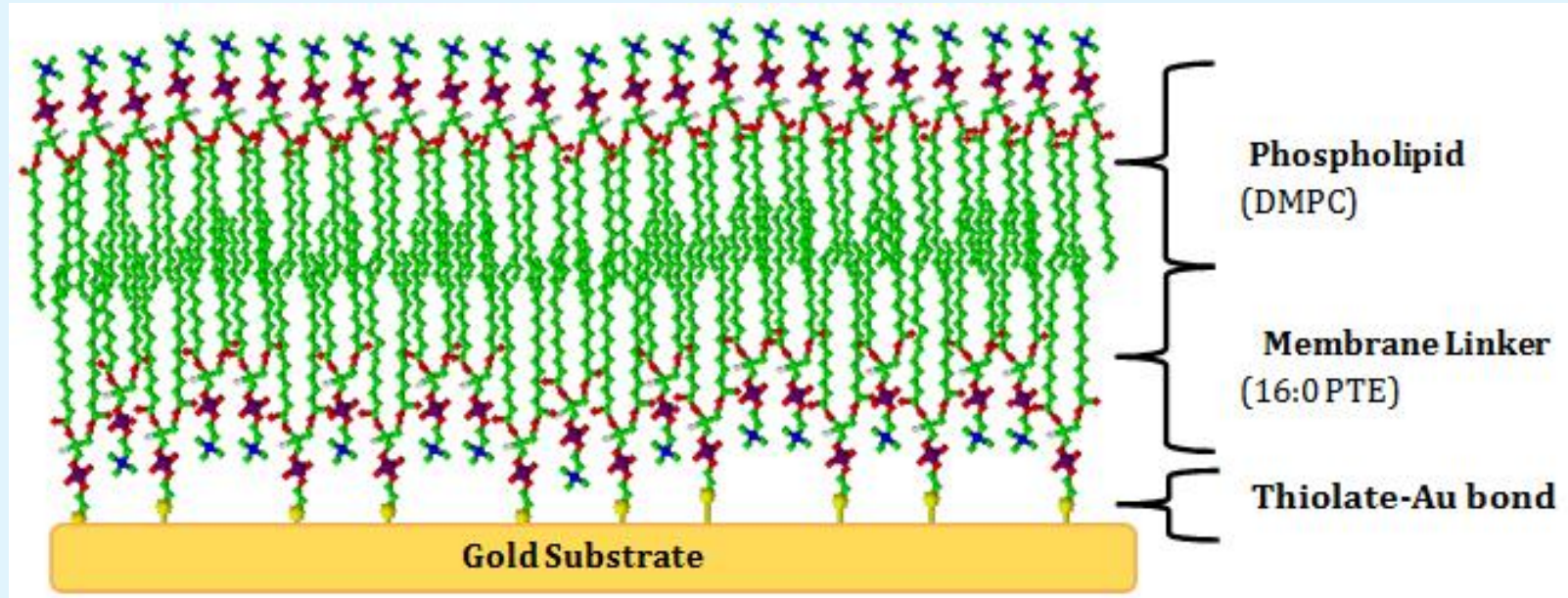

Figure 2: Schematic representation of a tethered bilayer lipid membrane in aqueous solution. 1,2-dipalmitoyl-snglycero-3-phosphothioethanol (16:0 PTE) with sulphur group bound to gold substrate causing the phospholipids to self-assemble around them, forming a bilayer with 16:0 PTE linkers.

Finally, h-BLM is more robust than s-LBM due to the strong interactions between the alkanethiol selfassembled monolayer (SAM) and the substrate [19]. Hybrid phospholipid membranes are still only a monolayer, mimicking only half the phospholipid bilayer and limiting the applications of transmembrane studies. In short, ideal properties of artificial membranes required for most studies are stability, reproducibility and durability. The artificial membrane must tolerate vigorous experimental techniques without degrading, enable transmembrane studies and have no substrate interferences. An artificial membrane that meets these specifications enables the use of electrochemical techniques for analytical purposes. $t-$ BLM are the only artificial membrane that meet all these requirements. In this mini review, we will evaluate tBLMs and discuss research using this artificial membrane and electrochemical techniques to uncover any visible gaps and parameters that have been overlooked or misinterpreted in research over the last 10 years.

\section{Discussion}

Tethered bilayer lipid membranes are a class of selfassembling biomimetic structures that provide a platform for a variety of biophysical experiments such as reconstituted membrane proteins, biosensors that range from the detection of biological agents to pharmaceutical screening [22], redox reactions [21], ion transport across the membrane [23], antigen/antibody binding [24] and more in diverse fields. Attachment to a conducting surface such as gold $(\mathrm{Au})$ allows t-BLMs to be monitored via electrochemical techniques [21].
Gold is commonly used as the substrate as it is chemically inert and can easily be modified by sulphur bearing reagents such as 1,2-dipalmitoyl-sn-glycero-3phosphothioethanol (16:0 PTE), which tethers the lipid bilayer to the $\mathrm{Au}$ substrate (Figure 2) enhancing the membrane stability $[12,25]$. Silicon based substrates are also commonly used and have proven useful results [22,26-29]. t-BLMs represent the most promising class of artificial membranes due to the increasing ability to mimic fundamental properties of natural cell membranes, including fluidity, electrical sealing and capability of hosting transmembrane proteins. The advantage of t-BLM is that unlike BLM and h-BLM, $t-$ BLM are stable for months and do not collapse when exposed to electrochemical techniques such as transmembrane potentials larger than $\pm 500 \mathrm{mV}$ and robust mechanical mixing [30]. The t-BLM model also creates minimal interaction with the substrate, the free space available allows the introduction of proteins [31] and they exhibit a higher lipid diffusion coefficient relative to SAMs [21]. The compelling properties, diversity and capability of tethered membranes have caused t-BLM to be considered the closest artificial mimic to real biological cells.

Even though artificial membranes are a highly used method to gain information about the phospholipid bilayer, they still have some unknowns in their research. The lack of information about biomimetic membranes comes from the possible effects off double bonds in the phospholipid tails.

Membrane fluidity lacks a precise definition but it refers to the mobility of various membrane components 
such as lateral diffusion and flexibility [32]. Fluidity of the phospholipid bilayer needs to be precisely regulated as various processes can be affected if membrane fluidity moves beyond its threshold level [32]. Lipid fluidity is heavily dependent on two factors, sterol content and the number of unsaturation in the fatty acid tails of the phospholipid molecules. Fully saturated phospholipids such as DMPC create a neat 'zig-zag' configuration that forms a highly ordered membrane of low fluidity [33]. Conversely, unsaturated phospholipids have a larger area per lipid molecule which forms a loosely packed, highly fluidic membrane [9]. The larger area is caused by the cis conformation creating 'kinks' in the fatty acid tails as the double bond is unable to rotate [9]. Even though the cis configuration is less stable it is still favoured by nature over trans in phospholipid membranes as cis ensures the ideal fluidity and permeability [34]. The optimal functionality of membranes can only be obtained by the bent structure of the cis configuration [34].

Increasing the amount of unsaturation in the phospholipid tails increases its area, and consequently increases membrane fluidity [35]; Figure 3 demonstrates this trend.

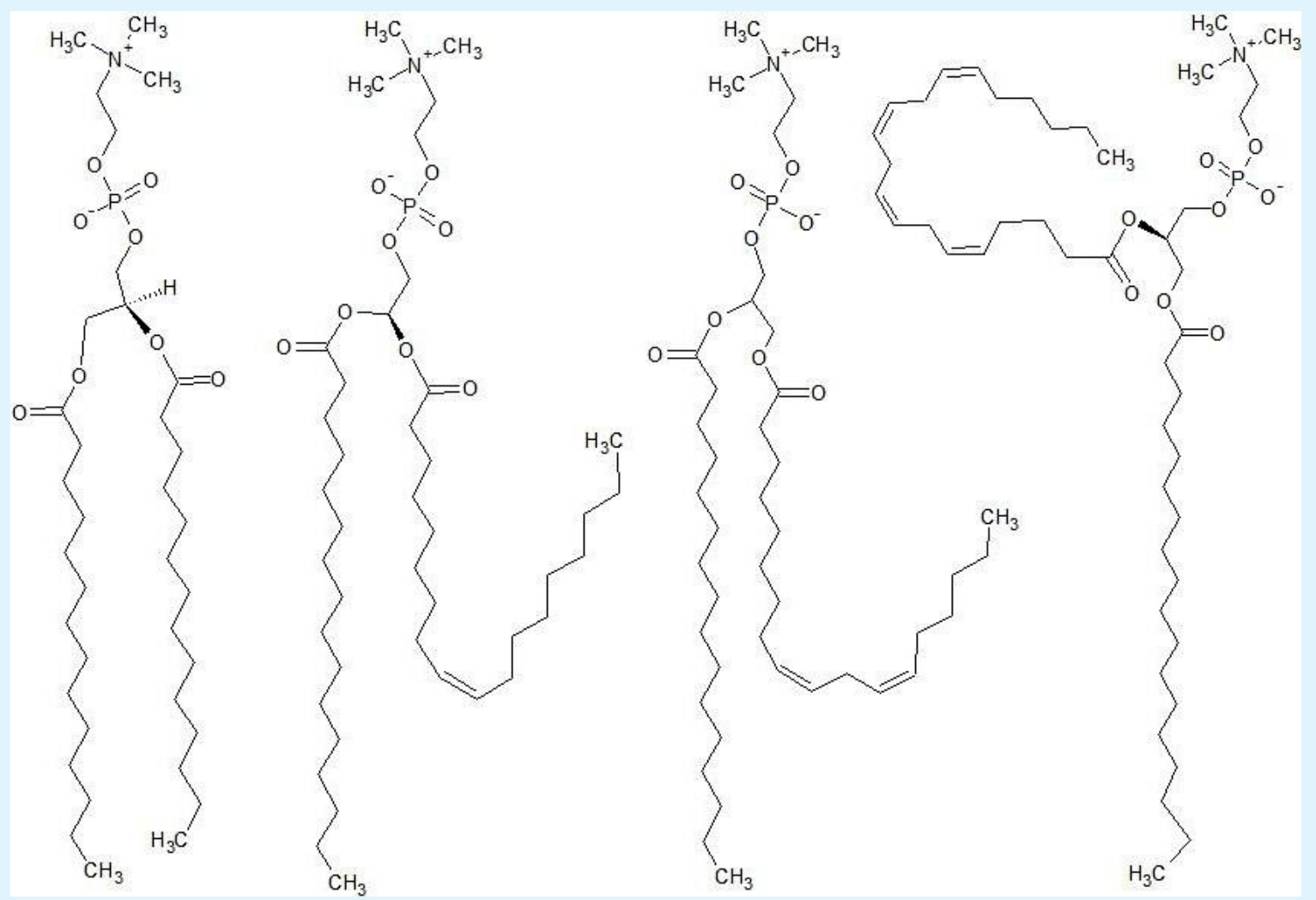

Figure 3: Molecular structures of (from left to right) 1,2-dimyristoyl-sn-glycero-3-phosphocholine (14:0/14:0), 1palmitoyl-2-oleoyl-sn-glycero-3-phosphocholine (16:0/18:1), 1-palmitoyl-2-linoleoyl-sn-glycero-3-phosphocholine (16:0/18:2) and 1-stearoyl-2-arachidonoyl-sn-glycero-3-phosphocholine (18:0/20:4). Increasing the number of double bonds in the fatty acid tails increases the severity of the kink.

Nature uses the number of unsaturation and sterol content to control fluidity of the membrane for environments with varied temperatures [36]. As temperature decreases saturated phospholipids are compressed together creating a dense ridged structure that is susceptible to solidification [37]. But, if unsaturated phospholipids are compressed the 'kinks' in the fatty acid tails maintain space between adjacent phospholipids sustaining membrane fluidity [37]. The temperature required to change the physical state of the phospholipids from ordered gel phase to disordered liquid phase depends on the transition temperature of the individual phospholipid type [38]. Sterols like cholesterol buffer the effects of temperature on fluidity by decreasing the activation energy for rotational diffusion [39]. A decrease in activation energy permits less change in fluidity per degree change in temperature establishing fluidic control of the membrane [40]. Both 


\section{Medicinal \& Analytical Chemistry International Journal}

cholesterol and unsaturation are important properties of a cellular membrane but these properties have not been simultaneously explored nor just exploration of unsaturation effect on the membrane behavior.

Unsaturated phospholipids are naturally occurring in biological membranes but the amount of saturated and unsaturated bonds varies depending on the type of cell and membrane. Approximately $35-45 \%$ of phospholipids are saturated and the remainders are unsaturated which contain between 1 and 6 cis double bonds [41]. Surprisingly, mostly fully saturated phospholipids such as 1,2-dimyristoyl-sn-glycero-3phosphocholine (PC(14:0/14:0)) and 1,2-dipalmitoyl$s n$-glycero-3-phosphocholine (PC(16:0/16:0)), are used to prepare t-BLMs [22-24,42]. The phospholipid 1palmitoyl-2-oleoyl-sn-glycero-3- phosphocholine (PC (16:0/18:1)) has also been used to conduct t-BLM studies $[29,43]$ which contains one cis double bond. The 1,2-dioleoyl-sn-glycero-3-ethylphosphocholine(EDOPC), containing two cis double bonds was only used in one study of t-BLMs [43]. The t-BLMs created in these various studies ( $\mathrm{t}$-BLMs composed $100 \%$ by only one phospholipid) are not accurate representations of real biological membranes, as real biological membranes possess many types of phospholipids with varied amounts of cis double bonds. Therefore, what we are reporting may not be an accurate representation of what is happening in a real cell membrane. We may need to rethink how mimetic artificial t-BLM truly are.

Membrane composition is the primary factor that has been overlooked in regards to t-BLM research. Due to the abundant types of membranes it will be a challenge to produce a t-BLM that truly mimics a biological plasma membrane. This challenge is currently incomplete and in some cases completely overlooked. Figure 4 demonstrates some composition varieties that have caused this challenge of creating a true biological mimic. Some of the most common forms of phospholipid head groups found in plasma membranes of mammalian cells are seen in Figure 4a. Cholesterol is also a very abundant lipid in plasma membranes with a molar ratio of 1.0 [44]. Due to its overall neutral charge and abundance [45] phosphatidylcholine (PC) head groups are commonly used for the entirety of research $[22-24,29,42,43]$ but what effect do the other head groups have on t-BLM behaviour? For example phosphatidylserine (PS) has an overall negative charge [46]; how does this affect substrate-membrane interaction in a t-BLM? The same principle applies to the variation in unsaturation levels in phospholipid tails and its effects on membrane behaviour. Simply using PC head groups and fully saturated lipid tails to build a tBLM is not an accurate enough biological mimic.
A)

\section{PHOSPHOLIPID HEAD GROUPS}
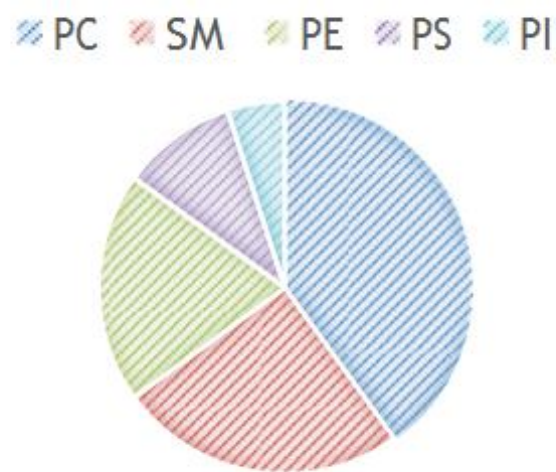

B)

\section{PHOSPHOLIPID TAIL UNSATURATION}

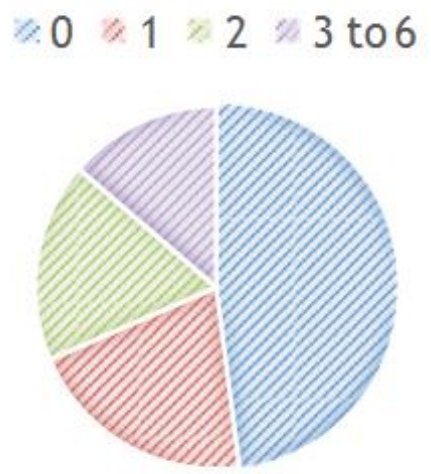

Figure 4: Approximate percentage composition for mammalian cell of: A) phospholipid head groups; phosphatidylcholine (PC): 40\%, phosphatidylethanolamine (PE): 21\%, phosphatidylinositol (PI): 6\%, phosphatidylserine (PS): $9 \%$ and sphingomyelin (SM): 24\%. B) phospholipid tail unsaturation; 0 double bonds: $47 \%$, 1 double bond: $21 \%$, 2 double bonds: $18 \%$ and 3-6 double bonds: $14 \%$. Data retrieved from $[44,47,48]$. 
Electrochemical Impedance Spectroscopy (EIS) is a common technique used to monitor the conductivity and dielectric properties of t-BLM [26,43,49-52]. EIS is the only technique that allows the simultaneous visualisation of conductivity and resistance. Two properties that is vital to the understanding and creation of t-BLM. Conductivity incorporates the exploration of capacitance including change in height and investigation of dielectric properties of the membrane [53]. The lipid bilayer composition of cells can modify the capacitance and resistance values [26], and also alter permeation of molecules into the cells [54]. For one study using 1-palmitoyl-2-oleoyl-snglycero-3-phosphocholine and 1,2-dioleoyl-sn-glycero3- ethylphosphocholine, containing one cis double bond and 2 cis double bonds respectively, a slight change in conduction and a small amount of variation in capacitance was observed [43]. It was concluded that the main determinant for this change is the hydrogen bonding between the phosphate oxygen molecules on adjacent phospholipids when varying $\mathrm{H}_{3} \mathrm{O}^{+}$ concentration [43]. But what hasn't been considered is the possible effect of double bonds on the electrical properties, which may account for some change observed in this study. By adding unsaturation into the phospholipid tails and possible other membrane components the resistance and capacitance might consequently change but to what degree is still unknown. The availability of this information is required to set new standards for the electrical properties of $\mathrm{t}$-BLMs that are known today.

Unsaturation in newly synthesised tether molecules has been explored [22]. The study's focus was to identify if the newly created tether molecules could support the formation of t-BLMs, which was concluded possible. The results from unsaturated tether molecules were compared to those of fully saturated tether molecules. However, by also using unsaturated phospholipids in the t-BLM setup it cannot be confirmed whether changes in membrane behaviour is the cause of the unsaturation in the tethers, the variation in tether lengths or the unsaturation in the phospholipid tails [22]. This was a successful study as they did achieve the initial aim but in regards to membrane behaviour it is inconclusive what caused the changes in membrane properties, as there were many variations in the setup.

The ever-growing frequency of multi-drug resistant pathogens has specifically caught the interest of t-BLM studies [55]. The hunt for alternative antibiotic treatments and antimicrobial peptides (AMPs) has resulted in utilization of $\mathrm{t}$-BLMs to test the mechanism of action of new and commonly used pharmaceuticals. The largest problem found in majority of studies is the lack of similarity between a real biological membrane and the synthesized t-BLM [52]. The AMP Cecropin B has been tested on a t-BLM that was aimed to mimic a mammalian cell. However, one unsaturation in phospholipid tails and integration of cholesterol was used to produce the t-BLM [52]. The study states it relies on the changes in permeability but has not uncovered possible effects of unsaturation composition on permeability [52]. Another study testing Melittin, another AMP, on only fully saturated t-BLMs has also not considered the possible effect of unsaturation on membrane behaviour [56]. This is a common pattern seen in many studies using t-BLMs as membrane composition has not been considered a priority [57].

\section{Overlook}

Although numerous studies using t-BLMs have uncovered useful information, it is almost as if a critical step has been skipped. Jumping straight from the success of being able to create a t-BLM to testing varieties of things on or within them. Instead, perfection of the t-BLM should have been completed first before elaborate testing took place. This overlooked vital step has barred many studies from comparing their findings to real biological membranes, which one would assume is the ideal aim behind using artificial membranes in the first place. Commonly, it is stated that there is an advantage behind comparing a t-BLM study to other artificial membrane studies [58] but the overall aim is to compare these systems to real biological membranes not more artificial ones. The macrocosm idea behind an artificial membrane has been forgotten. Making this article more of a reminder that going back to basics strengthens the foundation of research.

\section{References}

1. Cremer C, Masters BR (2013) Resolution enhancement techniques in microscopy. The European Physical Journal H 38(3): 281-344.

2. Kwak KJ, Valincius G, Liao WC, Hu X, Wen X, et al. (2010) Formation and Finite Element Analysis of Tethered Bilayer Lipid Structures. Langmuir 26(23): 18199-18208.

3. Navratil T, Šestakova I, Dytrtova JJ, Jakl M, Marecek V (2009) Study of Charged Particles Transport across Model and Real Phospholipid Bilayers, In: M. Otesteanu, S. Celikyay, N. Mastorakis, S. Lache, F.K. Benra (Eds.), $7^{\text {th }}$ WSEAS International Conference on Environment, Ecosystems and Development, World Scientific and Engineering Academy and Society, pp: 212-217. 


\section{Medicinal \& Analytical Chemistry International Journal}

4. Singer SJ, Nicolson GL (1972) The Fluid Mosaic Model of the Structure of Cell Membranes. Science 175(4023): 720-731.

5. Malkia A, Liljeroth P, Kontturi K (2003) Membrane activity of ionisable drugs-a task for liquid-liquid electrochemistry? Electrochemistry Communicatio ns 5(6): 473-479.

6. Siontorou CG, Nikoleli GP, Nikolelis DP, Karapetis SK (2017) Artificial Lipid Membranes: Past, Present, and Future. Membranes 7(3): 38.

7. Li J, Wang X, Zhang T, Wang C, Huang Z, et al. (2015) A reviews on phospholipids and their main applications in drug delivery systems. Asian Journal of Pharmaceutical Sciences 10(2): 81-98.

8. Montealegre C, Verardo V, Luisa Marina M, Caboni MF (2014) Analysis of glycerophospho- and sphingolipids by CE. Electrophoresis 35(6): 779792.

9. Weijers RNM (2012) Lipid Composition of Cell Membranes and Its Relevance in Type 2 Diabetes Mellitus. Current Diabetes Reviews 8(5): 390-400.

10. Dod BJ, Gray GM (1968) The lipid composition of rat-liver plasma membranes. Biochimica et Biophysica Acta (BBA) - Biomembranes 150: 397404.

11. Eibl H (1980) Synthesis of glycerophospholipids. Chemistry and Physics of Lipids 26(4): 405-429.

12. Jackman JA, Knoll W, Cho NJ (2012) Biotechnology Applications of Tethered Lipid Bilayer Membranes. Materials 5(12): 2637-2657.

13. Sang-Kyu Lee LGCP, Sala RF, Holmes SP, Ryan KJ, Todd Becker (2005) Ion channel switch array: A biosensor for detecting multiple pathogens. Industrial Biotechnology 1(1): 26-31.

14. Jianrong C, Yuqing M, Nongyue H, Xiaohua W, Sijiao L (2004) Nanotechnology and biosensors. Biotechnology Advances 22(7): 505-518.

15. Stamatialis D, Papenburg B, Gironés $M$, Jabar $S$, Bettahalli S, et al. (2008) Medical applications of membranes: Drug delivery, artificial organs and tissue engineering. Journal of Membrane Science 308(1-2): 1-34.

16. Liu J, Jiang X, Ashley C, Brinker CJ (2009) Electrostatically Mediated Liposome Fusion and Lipid Exchange with a Nanoparticle-Supported Bilayer for Control of Surface Charge, Drug
Containment, and Delivery. Journal of the American Chemical Society 131(22): 7567-7569.

17. Bowry SK (2002) Dialysis membranes today. The International journal of artificial organs 25(5): 447460.

18. Winterhalter M (2000) Black lipid membranes. Current Opinion in Colloid \& Interface Science 5(34): 250-255.

19. Khan MS, Dosoky NS, Williams JD (2013) Engineering Lipid Bilayer Membranes for Protein Studies. International Journal of Molecular Sciences 14(11): 21561-21597.

20. Kuhner M, Tampe R, Sackmann E (1994) Lipid mono- and bilayer supported on polymer films: composite polymer-lipid films on solid substrates. Biophysical Journal 67(1): 217- 226.

21. Valincius G, Meškauskas T, Ivanauskas F (2012) Electrochemical Impedance Spectroscopy of Tethered Bilayer Membranes. Langmuir 28(1): 977990.

22. Budvytyte R, Valincius G, Niaura G, Voiciuk V, Mickevicius M, et al. (2013) Structure and Properties of Tethered Bilayer Lipid Membranes with Unsaturated Anchor Molecules. Langmuir 29(27): 8645-8656.

23. Robertson JW, Friedrich MG, Kibrom A, Knoll W, Naumann RL, et al. (2008) Modeling ion transport in tethered bilayer lipid membranes. The Journal of Physical Chemistry B 112(34): 10475-10482.

24. Spencelayh MJ, Cheng Y, Bushby RJ, Bugg TD, Li JJ, et al. (2006) Antibiotic action and peptidoglycan formation on tethered lipid bilayer membranes. Angewandte Chemie International Edition 45(31): 2111-2116.

25. Janshoff A, Galla HJ, Steinem C (2004) Biochemical Applications of Solid Supported Membranes on Gold Surfaces: Quartz Crystal Microbalance and Impedance Analysis, Planar Lipid Bilayers (BLM's) and Their Applications, Elsevier, Amsterdam, The Netherlands, pp: 991-1017.

26. Zhou W, Burke PJ (2017) Versatile Bottom-Up Synthesis of Tethered Bilayer Lipid Membranes on Nanoelectronic Biosensor Devices. ACS Applied Materials \& Interfaces 9(17): 14618-14632.

27. Junghans A, Köper I (2010) Structural Analysis of Tethered Bilayer Lipid Membranes. Langmuir 26(13): 11035-11040. 


\section{Medicinal \& Analytical Chemistry International Journal}

28. Flynn KR, Martin LL, Ackland ML, Torriero AAJ (2016) Real-time quartz crystal microbalance monitoring of free docosahexaenoic acid interactions with supported lipid bilayers. American Chemical Society 32(45): 11717-11727.

29. Wagner ML, Tamm LK (2000) Tethered polymersupported planar lipid bilayers for reconstitution of integral membrane proteins: silanepolyethyleneglycol-lipid as a cushion and covalent linker. Biophysical Journal 79(3): 1400-1414.

30. Cranfield CG, Cornell BA, Grage SL, Duckworth P, Carne S, et al. (2014) Transient Potential Gradients and Impedance Measures of Tethered Bilayer Lipid Membranes: Pore-Forming Peptide Insertion and the Effect of Electroporation. Biophysical Journal 106(1): 182-189.

31. Cornell BA, Krishna G, Osman PD, Pace RD, Wieczorek L (2001) Tethered-bilayer lipid membranes as a support for membrane-active peptides. Biochemical Society Transactions 29(Pt4): 613-617.

32. Lentz BR (1989) Membrane "fluidity" as detected by diphenylhexatriene probes. Chemistry and Physics of Lipids 50(3-4): 171-190.

33. Seelig A, Seelig J (1977) Effect of a single cis double bond on the structures of a phospholipid bilayer. Biochemistry 16(1): 45-50.

34. Ferreri C, Chatgilialoglu C (2015) Membrane lipidomics for personalized health, John Wiley and Sons, Inc., Chichester, West Sussex, Hoboken.

35. Yang X, Sheng W, Sun GY, Lee JCM (2011) Effects of fatty acid unsaturation numbers on membrane fluidity and $\alpha$-secretase-dependent amyloid precursor protein processing. Neurochemistry international 58(3): 321-329.

36. Xu X, London E (2000) The Effect of Sterol Structure on Membrane Lipid Domains Reveals How Cholesterol Can Induce Lipid Domain Formation. Biochemistry 39(5): 843-849.

37. Chapman D (2009) Phase transitions and fluidity characteristics of lipids and cell membranes. Quarterly Reviews of Biophysics 8(2): 185-235.

38. Cevc G (1991) How membrane chain-melting phase-transition temperature is affected by the lipid chain asymmetry and degree of unsaturation: an effective chain-length model. Biochemistry 30(29): 7186-7193.
39. Bittman R, Clejan S, Rottem S (1983) Transbilayer distribution of sterols in mycoplasma membranes: a review. The Yale Journal of Biology and Medicine 56(5-6): 397-403.

40. Cooper RA (1978) Influence of increased membrane cholesterol on membrane fluidity and cell function in human red blood cells. Journal of Supramolecular Structure 8(4): 413- 430.

41. Erman F, Aydin S, Demir Y, Akcay F, Bakan E (2006) Determination of Saturated and Unsaturated Fatty Acids Amount in Leukocyte Membranes from Subjects Fed with Solid and Fluid Oils. Journal of Biochemistry and Molecular Biology 39(5): 516521.

42. Vockenroth IK, Rossi C, Shah MR, Köper I (2009) Formation of tethered bilayer lipid membranes probed by various surface sensitive techniques. Biointerphases 4(2): 19-26.

43. Cranfield CG, Berry T, Holt SA, Hossain KR, Le Brun AP, et al. (2016) Evidence of the Key Role of H3O+ in Phospholipid Membrane Morphology. Langmuir 32(41): 10725-10734.

44. van Meer G, Voelker DR, Feigenson GW (2008) Membrane lipids: where they are and how they behave. Nature Reviews Molecular Cell Biology 9(2): 112-124.

45. Pasenkiewicz-Gierula M, Takaoka Y, Miyagawa $H$, Kitamura K, Kusumi A (1999) Charge Pairing of Headgroups in Phosphatidylcholine Membranes: A Molecular Dynamics Simulation Study. Biophysical Journal 76(3): 1228-1240.

46. McLaughlin S, Harary H (1974) Phospholipid FlipFlop and the Distribution of Surface Charges in Excitable Membranes. Biophysical Journal 14(3): 200-208.

47. van Meer G, de Kroon AI (2011) Lipid map of the mammalian cell. J Cell Sci 124: 5-8.

48. Ingólfsson HI, Melo MN, van Eerden FJ, Arnarez C, Lopez CA, et al. (2014) Lipid Organization of the Plasma Membrane. Journal of the American Chemical Society 136(41): 14554-14559.

49. Lin J, Szymanski J, Searson PC, Hristova K (2010) Effect of a Polymer Cushion on the Electrical Properties and Stability of Surface-Supported Lipid Bilayers. Langmuir 26(5): 3544-3548.

50. He L, Robertson JWF, Li J, Kärcher I, Schiller SM, et al. (2005) Tethered Bilayer Lipid Membranes Based 


\section{Medicinal \& Analytical Chemistry International Journal}

on Monolayers of Thiolipids Mixed with a Complementary Dilution Molecule. 1. Incorporation of Channel Peptides. Langmuir 21(25): 1166611672 .

51. Naumowicz M, Figaszewski ZA (2005) Impedance Analysis of Lipid Domains in Phosphatidylcholine Bilayer Membranes Containing Ergosterol. Biophysical Journal 89(5): 3174-3182.

52. Juhaniewicz J, Szyk-Warszyńska L, Warszyński P, Sęk S (2016) Interaction of Cecropin B with Zwitterionic and Negatively Charged Lipid Bilayers Immobilized at Gold Electrode Surface. Electrochimica Acta 204(20): 206-217.

53. Valincius G, Mickevicius M (2015) Tethered Phospholipid Bilayer Membranes: An Interpretation of the Electrochemical Impedance Response. Advances in Planar Lipid Bilayers and Liposomes Elsevier 21: 27-61.

54. Walter A, Gutknecht J (1986) Permeability of small nonelectrolytes through lipid bilayer membranes. The Journal of Membrane Biology 90(3): 207-217.
55. Bayramov D, Zhenghao L, Patel E, Izadjoo M, Hosan $\mathrm{K}$, et al. (2018) A Novel Peptide-Based Antimicrobial Wound Treatment is Effective Against Biofilms of Multi-Drug Resistant Wound Pathogens. Military Medicine 183(1): 481-486.

56. Juhaniewicz J, Sek S (2016) Interaction of Melittin with Negatively Charged Lipid Bilayers Supported on Gold Electrodes. Electrochimica Acta 197: 336343.

57. Krishna G, Schulte J, Cornell BA, Pace RJ, Osman PD (2003) Tethered Bilayer Membranes Containing Ionic Reservoirs: Selectivity and Conductance. Langmuir 19(6): 2294-2305.

58. Atanasov V, Knorr N, Duran RS, Ingebrandt $\mathrm{S}$, Offenhäusser A, et al. (2005) Membrane on a Chip: A Functional Tethered Lipid Bilayer Membrane on Silicon Oxide Surfaces. Biophysical Journal 89(3): 1780-1788. 